\title{
The Indicatrix of the Surface in Four-Dimensional Galilean Space
}

\author{
Artykbaev Abdullaaziz ${ }^{1}$, Nurbayev Abdurashid Ravshanovich, ${ }^{2, *}$ \\ ${ }^{1}$ Railway Engineering Institute, Uzbekistan \\ ${ }^{2}$ Department of Geometry and Topology, National University of Uzbekistan, Uzbekistan
}

Received February 19, 2020; Revised March 17, 2020; Accepted March, 28, 2020

Copyright $@ 2020$ by authors, all rights reserved. Authors agree that this article remains permanently open access under the terms of the Creative Commons Attribution License 4.0 International License

\begin{abstract}
This article discusses geometric quantities associated with the concept of surfaces and the indicatrix of a surface in four-dimensional Galileo space. In this case, the second orderly line in the plane is presented as a surface indicatrix. It is shown that with the help of the Galileo space movement, the second orderly line can be brought to the canonical form. The movement in the Galileo space is radically different from the movement in the Euclidean space. Galileo movements include parallel movement, axis rotation, and sliding. Sliding gives deformation in the Euclidean space. The surface indicatrix is deformed by the Galileo movement. When the indicatrix is deformed, the surface will be deformed. In the classification of three-dimensional surface points in the four-dimensional Galileo phase, the classification of the indicatrix of the surface at this point was used. This shows the cyclic state of surface points other than Euclidean geometry. The geometric characteristics of surface curves were determined using the indicatrix test. It is determined what kind of geometrical meaning the identified properties have in the Euclidean phase. It is shown that the Galilean movement gives surface deformation in the Euclidean sense. Deformation of the surface is indicated by the fact that the Gaussian curvature remains unchanged.
\end{abstract}

Keywords Principal Curvatures, Deformation, Galilean Space, Special Plane, Sliding, Surface Classification, Total Curvature, Cyclic Surface

\section{Introduction.}

While investigating a surface in space, it is important to know its geometric dimensions: the first and the second fundamental forms, the total and mean curvatures, and also the principal curvatures. The principal curvatures of the surface are clarified by its indicatrix at a given point. In order to classify the surface around the given point, it is important to classify its surface at that point.

The first and the second fundamental forms, the total and mean curvatures, and also the principal curvatures of the surface in three-dimensional Galilean space are determined in the [3],[8]. In this space the Frenet formulas have been formed in the [4] studies. And in this article, we are going to subdivide the points of the surface into classes with the help of the indecatrix of the surface.

Preliminiaries. Let $\vec{X}=\left(x_{1}, x_{2}, x_{3}, x_{4}\right)$ and $\vec{Y}=\left(y_{1}, y_{2}, y_{3}, y_{4}\right)$ vectors are defined on $A_{4}$ affine space. If scalar product is performed by

$$
\left\{\begin{array}{c}
x_{1} y_{1}, \text { if } \Rightarrow x_{1} y_{1} \neq 0 \\
x_{2} y_{2}+x_{3} y_{3}+x_{4} y_{4}, \text { if } \Rightarrow x_{1} y_{1}=0
\end{array}\right.
$$

the $A_{4}$ space is called four-dimensional Galilean space. From this [1] scalar product $\vec{X}=\left(x_{1}, x_{2}, x_{3}, x_{4}\right)$ the norm of the vector will be calculated as

$$
\left\{\begin{array}{c}
\left|x_{1}\right|, \text { if } \Rightarrow x_{1} \neq 0 \\
\sqrt{x_{2}^{2}+x_{3}^{2}+x_{4}^{2}}, \text { if } \Rightarrow x_{1}=0
\end{array} .\right.
$$

If two $M\left(a_{1}, a_{2}, a_{3}, a_{4}\right), N\left(b_{1}, b_{2}, b_{3}, b_{4}\right)$ points are represented, the distance between them can be determined by the following formula:

$$
\left\{\begin{array}{c}
\left|b_{1}-a_{1}\right|, \text { if } \Rightarrow a_{1} \neq b_{1} \\
\sqrt{\left(b_{2}-a_{2}\right)^{2}+\left(b_{3}-a_{3}\right)^{2}+\left(b_{4}-a_{4}\right)^{2}}, \text { if } \Rightarrow a_{1}=b_{1}
\end{array}\right.
$$

In Euclidean space, motions consist of parallel displacements and angles. The motion in three-dimensional Galilean space is 


$$
\left\{\begin{array}{c}
x^{\prime}=x+a \\
y^{\prime}=h_{1} x+y \cos \alpha+z \sin \alpha+b \\
z^{\prime}=h_{2} x-y \sin \alpha+z \cos \alpha+c,
\end{array}\right.
$$

$$
k_{1} x^{2}+k_{1} x^{2}+k_{1} x^{2}= \pm 1
$$

It can be defined as a canonical form.

Here $h_{1}, h_{2}$ are the angles of the Galilean space.

Proof. At first we take the equation (3) and put it to the equation (2). In this case

and in four-dimensional space, it is accomplished by substitution:

$$
\left\{\begin{array}{c}
t^{\prime}=t+a \\
x^{\prime}=h_{1} t+x \cos \alpha_{1}+y \cos \beta_{1}+z \cos \gamma_{1}+b \\
y^{\prime}=h_{2} t+x \cos \alpha_{2}+y \cos \beta_{2}+z \cos \gamma_{2}+c \\
z^{\prime}=h_{3} t+x \cos \alpha_{3}+y \cos \beta_{3}+z \cos \gamma_{3}+d
\end{array} .\right.
$$

$$
\begin{gathered}
\left(a_{11}+h_{1}^{2} a_{22}+h_{2}^{2} a_{33}+2 a_{12} h_{1}+2 a_{13} h_{2}\right) x^{2}+a_{22} y^{2} \\
+a_{33} z^{2}+2\left(a_{22} h_{1}+a_{12}\right) x y+2\left(a_{33} h_{2}+a_{13}\right) x z= \pm 1 .
\end{gathered}
$$

If $x y$ and $x z$ coefficients of multiplication in the equation are equal to 0

\section{Main Results}

While studying the surfaces, it is necessary to check them around the certain point. The surface can be monitored by making a surface indicatrix around the point of the surface.

We choose the point of the surface as the beginning of the coordinates, and at this point, we get the coordinate system such as OXYZ, in this case the surface indicatrix of the four-dimensional Galilean space $\left(\Gamma_{4}\right)$ will be the following type of the second order surface [5]:

$$
\begin{aligned}
& a_{11} x^{\prime \prime 2}+a_{22}^{\prime} y^{\prime \prime 2}+a_{33}^{\prime} z^{\prime \prime 2}+2 a_{12}^{\prime} x^{\prime \prime} y^{\prime \prime} \\
& +2 a_{13}^{\prime} x^{\prime \prime} z^{\prime \prime}+2 a_{23}^{\prime} y^{\prime \prime} z^{\prime \prime}= \pm 1 .
\end{aligned}
$$

If we make a special OYZ plane Euclidean rotation of this surface indicatrix [6] such as

$$
\left\{\begin{array}{c}
x^{\prime \prime}=x^{\prime} \\
y^{\prime \prime}=y^{\prime} \cos a+z^{\prime} \sin a \\
z^{\prime \prime}=-y^{\prime} \sin a+z^{\prime} \cos a
\end{array}\right.
$$

By the substitution, the coefficient of multiplication yz can be converted to 0 and the equation (1) will become

$$
a_{11} x^{\prime 2}+a_{22} y^{\prime 2}+a_{33} z^{\prime 2}+2 a_{12} x^{\prime} y^{\prime}+2 a_{13} x^{\prime} z^{\prime}= \pm 1 \text {. }
$$

For convenience, let's check indicatrix of the surface equation being considered as the form above (2).

Theorem 1. If the indicatrix equation of the surface (2) is $a_{22} \neq 0, a_{33} \neq 0$

$$
\left\{\begin{array}{c}
x^{\prime}=x \\
y^{\prime}=h_{1} x+y \\
z^{\prime}=h_{2} x+z
\end{array}\right.
$$

by the Galilean motion

$$
\left\{\begin{array} { l } 
{ a _ { 2 2 } h _ { 1 } + a _ { 1 2 } = 0 } \\
{ a _ { 3 3 } h _ { 2 } + a _ { 1 3 } = 0 }
\end{array} \Rightarrow \left\{\begin{array}{l}
h_{1}=-\frac{a_{12}}{a_{22}} \\
h_{2}=-\frac{a_{13}}{a_{33}}
\end{array} .\right.\right.
$$

We will clarify $h_{1}, h_{2}$. As it is clear in the form (6), it should be $a_{22} \neq 0, a_{33} \neq 0$.

If we put $h_{1}, h_{2}$, which is clarified in (6), to (5), the following formula will appear:

$$
\begin{array}{r}
\left(a_{11}+\frac{a_{12}^{2}}{a_{22}}+\frac{a_{13}^{2}}{a_{33}}-\frac{2 a_{12}^{2}}{a_{22}}-\frac{2 a_{13}^{2}}{a_{33}}\right) x^{2}+a_{22} y^{2}+a_{33} z^{2}= \pm 1 \\
\left(a_{11}-\frac{a_{12}^{2}}{a_{22}}-\frac{a_{13}^{2}}{a_{33}}\right) x^{2}+a_{22} y^{2}+a_{33} z^{2}= \pm 1 .
\end{array},
$$

If we insert the following designation

$$
\left\{\begin{array}{c}
k_{1}=a_{11}-\frac{a_{12}^{2}}{a_{22}}-\frac{a_{13}^{2}}{a_{33}} \\
k_{2}=a_{22} \\
k_{3}=a_{33}
\end{array}\right.
$$

We will get the equality (4). The theorem has been proven.

Accordingly, in order to move from the indicatrix equation of the surface (2) to the canonical form (7), it is necessary to make a Galilean motion: 


$$
\left\{\begin{array}{c}
x^{\prime}=x \\
y^{\prime}=-\frac{a_{12}}{a_{22}} x+y \\
z^{\prime}=-\frac{a_{13}}{a_{33}} x+z
\end{array}\right.
$$

Generally, with the help of the Galilean motion, a certain surface moves to this type of the surface. Since it hasn't been impacted on the form of the surface, we can possibly determine its classification by this movement.

To classify the surface at the given point in $\Gamma_{4}$, we will make the classification of its indicatrix.

I If the coefficients of the indicatrix are $a_{22} \neq 0, a_{33} \neq 0$, let's take the canonical form (7) of the indicatrix equation.

1) If $k_{1}, k_{2}, k_{3}$ have the same signs - the indicatrix is ellipsoid, and the point of the surface is elliptical.

2) If $k_{1}, k_{2}, k_{3}$ don't have the same signs, the indicatrix is one or two-sheet hyperboloid, and the point of the surface is hyperbolic.

3) If $k_{1}=0, k_{2} k_{3}>0$ - the indicatrix is an elliptical cylinder, and the point of the surface is parabolic.

4) If $k_{1}=0, k_{2} k_{3}<0$ - the indicatrix is an hyperbolic cylinder, and the point of the surface is parabolic.

II a) If the indecatrix coefficients are $a_{22} \neq 0, a_{33}=0, a_{13} \neq 0$, we will take the canonical form of the indicatrix equation (2). To check it, let's make the following Galilean transformation:

$$
\left\{\begin{array}{c}
x^{\prime}=x \\
y^{\prime}=-\frac{a_{12}}{a_{22}} x+y \\
z^{\prime}=\frac{a_{12}^{2}-a_{11} a_{22}}{2 a_{22} a_{13}} x+z
\end{array} .\right.
$$

In this Galilean substitution the equation of the surface (2) will be

$$
a_{22} y^{2}+2 a_{13} x z= \pm 1
$$

It is one or two-sheet hyperboloid. If we consider this surface is $X Z=\frac{1}{2 a_{13}}$ which is $y=0$ plane with its cross section on $O X Z$ plane, it can be the cyclic form in three-dimensional Galilean space [3]. If the indicatrix of the surface is $a_{22}>0$, it is one-sheet, and if it is $a_{22}<0$ - then it is two-sheet hyperboloid, the point of four-dimensional surface is a cyclic point.

b) If the indicatrix coefficients are $a_{22} \neq 0, a_{33}=0, a_{13}=0$, we will take the canonical form of the indicatrix equation. To check it, let's make a Galilean transformation.

$$
\left\{\begin{array}{c}
x^{\prime}=x \\
y^{\prime}=-\frac{a_{12}}{a_{22}} x+y \\
z^{\prime}=z
\end{array}\right.
$$

In this Galilean substitution the equation of the surface (2) will be

$$
\frac{a_{11} a_{22}-a_{12}^{2}}{a_{22}} x^{2}+a_{22} y^{2}= \pm 1 \text {. }
$$

If we take $\Delta=a_{11} a_{22}-a_{12}^{2}$, it is an elliptical cylinder in $\Delta>0$, a hyperbolic cylinder in $\Delta<0$, and in $\Delta=0 \quad y= \pm \frac{1}{\sqrt{a_{22}}}$ is a parallel plane.

The formers of the cylinders above are parallel to a special plane.

III If the indicatrix coefficients are $a_{22}=0, a_{33} \neq 0, a_{12} \neq 0 \quad$ and $a_{22}=0, a_{33} \neq 0, a_{12}=0$, the classification is similar with "II a)" and "II b)". Because this cases are the substitutions of the coordinate axes on the initial OYZ special plane.

IV IV. If the indicatrix coefficients are $a_{22}=0, a_{33}=0$, the equation form will be

$$
a_{11} x^{2}+2 a_{12} x y+2 a_{13} x z= \pm 1 .
$$

If we check the inticatrix equation of the surface with the form $y=-\frac{a_{11} x+2 a_{13} z}{2 a_{12}} \pm \frac{1}{2 a_{12} x}$ if it is $a_{12} \neq 0$, and with the form $z=-\frac{a_{11} x+2 a_{12} y}{2 a_{13}} \pm \frac{1}{2 a_{13} x}$, if it is, the indicatrix will have two asymptotes. One of them is $O Y Z$ 
special plane. So it is a hyperbolic cylinder.

If $a_{12}=0, a_{13}=0$, it means there are 2 parallel special planes with the equation $x^{2}= \pm \frac{1}{a_{11}}$.

If we rotation the indicatrix of the surface at a given point with the help of Galilean transformation, the surface will also be deformed.

Theorem. If we rotation the indicatrix of the surface at a given point with the help of Galilean transformation, the curvature of the surface at this point will be stayed the same.

Proof. To demonstrate a total curvature of the surface, it is important to indicate that the multiplication of the principal curvatures can also stay the same. The principal curvatures are equal to the reverse value of the semi-axes quadrate of the indicatrix at the given point of the surface. So to save the total curvature at the given point of the surface, the multiplication of the half axes of the indicatrix at the given point of the surface has to be saved.

Taking the indicatrix of the surface in Galilean space as the form (2), let's find its specific numbers.

$$
\left|\begin{array}{ccc}
a_{11}-\lambda & a_{12} & a_{13} \\
a_{12} & a_{22}-\lambda & a_{23} \\
a_{13} & a_{23} & a_{33}-\lambda
\end{array}\right|=0 .
$$

$\lambda_{1}, \lambda_{2}, \lambda_{3}$ which are the solutions of this equation, are the specific numbers. The semi-axes of the second order surface are equal to $\frac{1}{\sqrt{\left|\lambda_{1}\right|}}, \frac{1}{\sqrt{\left|\lambda_{2}\right|}}, \frac{1}{\sqrt{\left|\lambda_{3}\right|}}$. So we will compare the multiplications of the specific numbers derived from the equation after deformation and the numbers from the initial indicatrix equation.

It is evident from the determinant (11), that

$$
\lambda_{1} \lambda_{2} \lambda_{3}=\left|\begin{array}{ccc}
a_{11} & a_{12} & a_{13} \\
a_{12} & a_{22} & 0 \\
a_{13} & 0 & a_{33}
\end{array}\right|,
$$

the equality is relevant.

And now if we make a substitution (3) on the surface (2), the equation will be as the form (5). Let's make the following equation to find the specific numbers of its matrix.

$$
\left|\begin{array}{ccc}
a_{11}+h_{1}^{2} a_{22}+h_{2}^{2} a_{33}+2 a_{12} h_{1}+2 a_{13} h_{2}-\lambda^{\prime} & a_{22} h_{1}+a_{12} & a_{33} h_{2}+a_{13} \\
a_{22} h_{1}+a_{12} & a_{22}-\lambda^{\prime} & 0 \\
a_{33} h_{2}+a_{13} & 0 & a_{33}-\lambda^{\prime}
\end{array}\right|=0
$$

The solutions of this equation are the specific numbers of the surface in the form (5). The multiplication of these specific numbers are equal to

$$
\lambda_{1}^{\prime} \lambda_{2}^{\prime} \lambda_{3}^{\prime}=\left|\begin{array}{ccc}
a_{11}+h_{1}^{2} a_{22}+h_{2}^{2} a_{33}+2 a_{12} h_{1}+2 a_{13} h_{2} & a_{22} h_{1}+a_{12} & a_{33} h_{2}+a_{13} \\
a_{22} h_{1}+a_{12} & a_{22} & 0 \\
a_{33} h_{2}+a_{13} & 0 & a_{33}
\end{array}\right| .
$$

To calculate this determinant, we will use its alternatives [7].

Multiply the second row of the determinant to $-h_{1}$, add the result to the first row. Also multiply the third row to $-h_{2}$, add it to the first row. The final result will be

$$
\lambda_{1}^{\prime} \lambda_{2}^{\prime} \lambda_{3}^{\prime}=\left|\begin{array}{ccc}
a_{11}+a_{12} h_{1}+a_{13} h_{2} & a_{12} & a_{13} \\
a_{22} h_{1}+a_{12} & a_{22} & 0 \\
a_{33} h_{2}+a_{13} & 0 & a_{33}
\end{array}\right| .
$$

Now let's calculate the last case for the columns.

Multiply the second column of the determinant to $-h_{1}$, add the result to the first column. Also multiply the third column to $-h_{2}$, add it to the first column. The final result will be

$$
\lambda_{1}^{\prime} \lambda_{2}^{\prime} \lambda_{3}^{\prime}=\left|\begin{array}{ccc}
a_{11} & a_{12} & a_{13} \\
a_{12} & a_{22} & 0 \\
a_{13} & 0 & a_{33}
\end{array}\right| .
$$

As you can see, the multiplication of the specific numbers - $\lambda_{1} \lambda_{2} \lambda_{3}=\lambda_{1}^{\prime} \lambda_{2}^{\prime} \lambda_{3}^{\prime}$ are saved. So the multiplication of the semi-axes, the multiplication of the principal curvatures and the total curvatures at the given point of the surface are equal. The theorem has been proven.

The result. There are some surfaces in Euclidian space, which are not isometric and have the similar total curvatures at the given point.

Proof. As we know, the Galilean substitution does not save an isometry in Euclidian space. The surface moves to another surface, which is not isometric to itself. [2]

It is evident from all above, that the Galilean substitution does not impact on the total curvature at the point of the surface. So in Euclidian space the Galilean substitution transforms the surface to another surface, which is not symmetric, but has similar total curvatures at the given point.

\section{Conclusions}

The motion determinant in Galilean space is equal to 1, however, it is not orthogonal and symmetric. If we consider the system of the coordinates in four-dimensional Galilean space as $O X_{1} X_{2} X_{3} X_{4}$, here we call $O X_{1} X_{2} X_{3}$ as a hyperplane. In this motion, the points of the special plane of the surface are not changed. Any other point slides to the 
point $\left(t, x+h_{1}, y+h_{2}, z+h_{3}\right)$ on the hyperplane which goes by this point $(t, x, y, z)$. In this motion the isometry is not saved. Even if the is not saved, as it has been shown above, the full curvature is saved.

\section{REFRENCES}

[1] A.V.Khachaturyan “Geometriya Galileya” Moskva 2005.

[2] A.Artykbaev, B.M.Sultanov "Invariants of surface Indicatrix in a Special linear transformation" Matematics and Statistics 7(4):106-115.2019.

[3] Artikbayev A.,Sokolov D.D "Geometriya v tselom v ploskom prostranstve -vremeni” Tashkent, Fan,1991.180

[4] Erjavec, Z., Divjak, B., Horvat, D., The General Solutions of frenet's System in the Equiform Geometry of the Galilean, Pseudo-Galilean, Simple Isotropic and Double Isotropic Space, International Mathematical Forum, 6(2011), no. 17,837-856.

[5] A.R.Nurbayev, "Indikatrisa poverkhnostey v chetyrekhmernom galileyevom prostranstve" Mezhdunarodnoy konferentsii «Sovremennaya geometriya i yeye prilozheniya-2019» Kazan' 4 - 7 sentyabrya 2019 y.

[6] Vladimir Serdarushich “Analytic Geometry” CreateSpace Independent Publishing Platform, 2015. 174 pg.

[7] Leslie Hogben. Handbook of linear Algebra. Second edition. Lowa State university Ames, USA 2014.

[8] Kurbonov. E.K. About the surface of Galilean space. Uzbek Mathematical Journal, №.1, 46-52. 2005 y. (in russian).

[9] Sultanov. B.M. Exitence of a cyclic surface by a given function of total curvature. Acta NUUz, №. 2/2, 201-204. 2017.

[10] Artykbayev A. Classification of surface points in Galilean space. Research on the theory of surface in manifolds of sign-constant curvature. L., 1-15. 1987 y. (in russian).

[11] Dea Won Yoon. Some classification of translation surface in Galilean 3-space. Int. Journal of Math. Analysis, Vol. 6, No. 25-28, 1355-1361.2012 y. 